\title{
Il disagio femminile di una mamma lavoratrice, accompagnato dalla patologia di ADPKD, diventa più pungente e si fa sentire
}

Giornale di Tecniche Nefrologiche e Dialitiche 2018, Vol. 30(I) 30

(C) The Author(s) 2018

Reprints and permissions:

sagepub.co.uk/journalsPermissions.nav

DOI: I0.1 I77/03949362।8766527

journals.sagepub.com/home/gtn

\section{La voce dei pazienti}

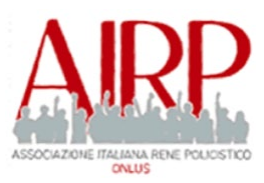

\section{Elisabetta ci scrive:}

Mi chiamo Elisabetta, ho 43 anni e ho l'ADPKD, ho un'unica figlia di anni 13 in sospetto ADPKD.

Mio padre già deceduto dopo soli 6 mesi di dialisi a 67 anni e mio zio morto per aneurisma cerebrale a circa 55 anni.

Alla visita per riconoscimento invalidità civile mi è stato riconosciuto, in seconda battuta, il $75 \%$ avendo anche una malformazione cardio-circolatoria.

Io sono in "buona salute", in terapia antipertensiva e lavoro a tempo pieno come dipendente pubblico. Non ho ancora manifestato l'insufficienza renale, sono al primo stadio, ma devo evitare sforzi fisici e cercare di cambiare stile di vita dal momento che cure farmacologiche non $\mathrm{mi}$ sono state proposte.

Un giorno dello scorso novembre 2016, dopo una giornata di lavoro arrivata a casa, avverto un dolore atroce acuto al fianco destro tanto da farmi respirare a singhiozzo ed ematuria. Dopo questo episodio a gennaio 2017 ho chiesto al lavoro la trasformazione del contratto da tempo pieno a part-time e, dal momento che concorsi o bandi per part-time escono molto di rado, potrei chiedere la trasformazione del rapporto di lavoro, secondo l'art. 8 del DL 15/06/2015 n. 81 del Jobs Act, che al punto 3 cita che "i lavoratori pubblici o privati affetti da gravi patologie cronico-degenerative ingravescenti, per i quali residui una ridotta capacità lavorativa $[\ldots]$, hanno diritto alla trasformazione del rapporto di lavoro [...]." A seguito della visita medico collegiale per la verifica dei requisiti per l'applicazione del D.L n. 81, la commissione si esprime con parere negativo e dichiara che non sussistono $\mathrm{i}$ requisiti per l'applicazione dell'art. 8 di tale decreto. Al lavoro quindi in un primo momento hanno rifiutato la mia domanda di part-time di 24 ore dicendomi che avrebbero concesso il part time solo se avessi accettato il turno notturno. La stanchezza, neanche a dirlo, è più forte che mai e anche se il mio fisico lo percepisco con almeno 10 anni in più, prego sempre affinché la mia testa rimanga lucida, attiva e giovane.

Dopo un anno di trattativa il part-time mi è stato concesso dallo scorso ottobre 2017 con 30 ore settimanali al posto di 36 , e per la durata di tre anni, come se io fra tre anni guarirò dall'ADPKD (magari!!!). Il medico di base mi dice di rivolgermi all'avvocato. Io chiedo un part-time adesso dopo 12 anni di servizio a tempo pieno, non ho mai rifiutato alcun incarico, mi sono sempre arrangiata con mia figlia e le mie assenze per "malattia" sono state molto poche visto che meno mi ammalo e meno farmaci prendo, meglio è.

L'ADPKD è parte integrante della nostra famiglia, è ancora una malattia nascosta e, poiché ad essere lesi sono organi interni invisibili, anche i tuoi diritti sono invisibili: sei considerata "sana" e questo è un' arma a doppio taglio perché da una parte c'è la tua privacy, la tua vita "normale", ma dall'altra i segni e i sintomi che si possono manifestare li senti solo tu, vedi la stanchezza fuori dal normale che ormai mi sta condizionando anche la mia vita sociale, perché ho solo, e devo avere, energie per il lavoro e per mia figlia, il subdolo dolore ai fianchi, l'ipertensione.

Sono donna e il lavoro che ti trovi ad affrontare ogni giorno è doppio e, se anche non avessi diritti, l'ADPKD è con me da quando sono nata, esiste, è una patologia a tutti gli effetti, che in prima persona cerchi di dimenticare ma lei non te lo permette.

Ogni giorno mi rivolgo ai due padri che ho in cielo affinché la ricerca vada avanti e che si trovi il farmaco giusto soprattutto per coloro i quali hanno figli che potrebbero esserne affetti e quindi averne bisogno.

Grazie per l'ascolto, Elisabetta 